\title{
WEB 2.0 SOLUTIONS TO WICKED CLIMATE CHANGE PROBLEMS
}

\author{
Alanah Kazlauskas \\ School of Business and Informatics (NSW), \\ Australian Catholic University, Sydney, Australia \\ Email: Alanah.Kazlauskas@acu.edu.au \\ Helen Hasan \\ School of Economics, University of Wollongong \\ Wollongong 2522, Australia \\ Email: hasan@uow.edu.au
}

\begin{abstract}
One of the most pressing 'wicked problems' facing humankind is climate change together with its many interrelated environmental concerns. The complexity of this set of problems can be overwhelming as there is such diversity among both the interpretations of the scientific evidence and the viability of possible solutions. Among the social technologies associated with the second generation of the Internet known as Web 2.0, there are tools that allow people to communicate, coordinate and collaborate in ways that reduce their carbon footprint, potentially becoming part of the climate change solution. However the way forward is not obvious or easy as Web 2.0, while readily accepted in the chaotic social world, is often treated with suspicion in the more ordered world of business and government. This paper applies a holistic theoretical sense-making framework to research and practice on potential Web 2.0 solutions to climate change problems. The suite of issues, activities and tools involved are viewed as an ecosystem where all elements are dynamic and interrelated. Through such innovative thinking the Information Systems community can make a valuable contribution to a critical global problem and hence find a new relevance as part of the solution.
\end{abstract}

\section{INTRODUCTION}

Many of the big challenges of the current digital age come from 'wicked problems'. Such problems are ill-defined, shifting definitions and multiple elements with conflicting objectives that demand resolution through a complex, holistic perspective (Rittel \& Webber. 1975, Courtney 2001). A typical example of a wicked problem, and arguably one of the most pressing challenges facing humankind, is climate change which comes with a whole raft of interrelated environmental concerns: Water, food, land degradation, species extinction, population growth, pollution etc (see eg Gore 2006, Garnaut 2008, Stern 2008). The pervasiveness of digital information and communications technologies (ICT) in human activity makes it appropriate that the concept and reality of the recent popularity of Web 2.0 be considered in the climate change debate. While efforts are being made to develop greener IT solutions, what has not been given sufficient 
consideration is the capability of Web 2.0 to support collaboration anywhere and anytime. However, in the context of formal human enterprises, businesses and governments, the social nature of these technologies challenges traditional ways of working. With the imperative to reduce our carbon footprint, surely Web 2.0 technologies provide an opportunity for the Information Technology (IT) and Information Systems (IS) communities to make a significant contribution to the challenge of addressing climate change. There is an imperative from considerations of performance, cost, social wellbeing and environmental sustainability for Web 2.0 and associated digital ecosystems to be a critical part of the climate change solution (Ghose et al 2008). To deal with the complex climate change situation within the context of a wicked problem, suitably diverse, creative and flexible approaches are needed for research in this dynamic area.

This paper begins with a theoretical sense-making framework that is later used to explore a complex holistic approach to research and practice as it is relative to ICT issues in the climate change problem. Firstly, the notion of an 'ecosystem' is introduced through a relevant example and used to conceptualise the current environment in a holistic, dynamic way that is appropriate for the study of wicked problems and facilitates the finding of innovative solutions. This provides a suitable perspective for the use of the complexity informed Cynefin framework as an analytical lens through which the problems of climate change and possible Web 2.0 solutions can be understood and advanced.

\section{SYSTEMS AND ECOSYSTEMS: A THEORETICAL PERSPECTIVE}

The concept of a system, based on general systems theory (Von Bertalanffy 1967), is central to several fields of study, e.g. systems thinking, systems dynamics and information systems (IS). A system can be described a as a purposeful collection of interrelated elements and is more than just a sum of the parts. Computer-based information systems have been essential to organisations since they appeared in the 1960s. They process transactions where data inputs are converted into outputs which include information. Most are ordered, predictable, planned, designed and tested to be fit for purpose. However, life and its problems are not always that straightforward.

The term 'ecosystem' offers a different way of conceptualising a problem space that is both emergent and organic. Appropriated from biology, an ecosystem denotes organisms, their environment and the many dynamic relationships and connections between them. The term is now widely used to describe systems in other fields that have became more complicated, less predictable and context dependent, with softer components such as human factors and organisational culture. In non-biological fields, ecosystems challenge traditional approaches to research as they defy most common empirical methods of data collection and analysis which seek to determine cause and effect or to create replicable models of a system. In practice it is difficult to know how to act purposefully in an ecosystem when everything is interrelated making it impossible to predict all the ramifications of an intervention. A digital ecosystem can be thought of as a suite of technological tools together with social and other contextual elements that interact as part of a complex but meaningful whole. It is clear that different types of systemic problems require different levels of understanding and analysis as well as different types of solutions.

To make sense of the whole spectrum of systems and ecosystems we advocate the use of Cynefin (pronounced kun-ev'in) framework developed through research and practice of Knowledge Management (KM) by Dave Snowden (2002) when working at IBM. 


\section{THE CYNEFIN FRAMEWORK FOR RESEARCHING ECOSYSTEMS}

Cynefin is a holistic, sense-making framework that draws on complexity theory to provide a perspective, language and conceptual lens that allows us to characterise problems and find suitable solutions. As shown in Figure 1, the Cynefin framework has five domains reflecting the different relationships between cause and effect and different ways of working in the various domains. Each domain has a different mode of community behaviour and each implies the need for a different form of management and a different leadership style with the adoption of different tools, practices and conceptual understanding. Four of the Cynefin domains set the possible contexts for collective decision making, an approach which has been used in knowledge management as well as in other applications including conflict resolution. These four domains starting at the bottom right then moving anticlockwise are:

The Known or Simple Domain, in which the relationship between cause and effect is obvious to all. The approach suited to this context is to Sense - Categorise - Respond (SCR). This suits a centralised bureaucratic way of working using vertical command and control with weak horizontal links in organisations. Solutions to problems in this domain often involve the generation of best practice, standard routines, rules and regulations.

The Knowable or Complicated Domain, in which the relationship between cause and effect requires analysis or some other form of investigation and/or the application of expert knowledge. The approach here is to Sense - Analyse - Respond (SAR). This domain is the realm of scientific research where it is assumed that all knowledge is knowable. Matrix organisational structures reside in this domain with strong relationships both vertically and horizontally.

The Unordered Complex Domain, in which the relationship between cause and effect can only be perceived in retrospect, not in advance. The approach is to Probe - Sense - Respond (PSR) and then allow emergent practice. Aspects of Complexity Theory developed in biology are relevant to this domain. Community and networked structures are usually here. Solutions to problems in this domain are, in the main, the subject of this paper.

The Chaotic Unordered Domain, in which there is no relationship between cause and effect at systems level. The approach is to Act - Sense - Respond (ASR) to discover novel practice. Aspects of Chaos Theory developed in mathematical disciplines are relevant to this domain. The connections between individuals and organisations working in this domain are weak. Here there is no discernable structure or obvious solutions.

The two right hand domains (known/simple and knowable/complicated) are ordered whereas those on the left (complex and chaos) are sensibly viewed as unordered. As ordered or simple problems become more complicated we can either endeavour retain order by simplifying and decomposing into small problems that can be tackled more easily or we can move to the left side of Cynefin and take a holistic view where the complexity and chaos is retained. Wicked problems, that defy obvious solutions or have conflicting objectives are in the unordered domains and need to be dealt with as such.

The fifth central domain is disorder, which is the destructive state of not knowing what type of causality exists and thus not knowing which way of working is best. While problems may legitimately be allowed to exist in the other four domains if approached with suitable solutions, 
those in states of disorder are normally harmful and should be guided into one of the other domains. Space constraints do no allow disorder to be addressed in this paper nor is it particularly relevant.

\section{Unordered Domains $\quad$ Ordered Domains}

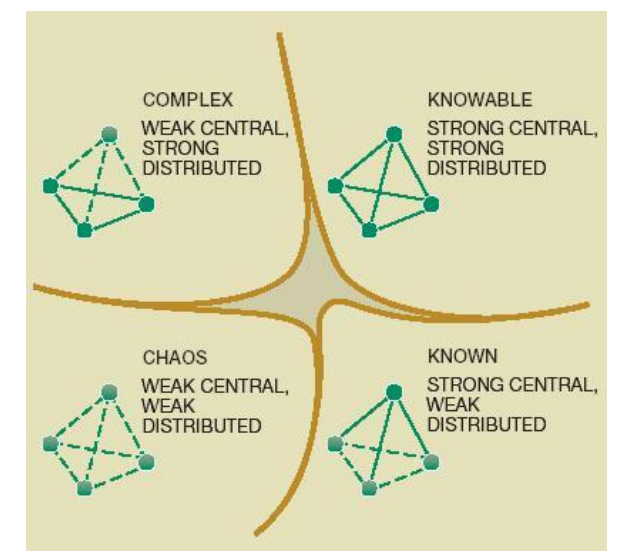

Figure 1: The Cynefin framework with two ordered and two unordered domains with disorder in the centre. The connection strengths of Cynefin domains are drawn from Kurtz and Snowden

(2003).

In proposing the Cynefin model, initially for KM but increasingly for other areas of investigation, Snowden (2002) makes a point of strongly resisting the existence of a single or idealised model and raises an awareness and understanding of the borders between different domains and the acquisition of tools and techniques to enable border transitions when needed. People are usually most comfortable in one of the Cynefin domains and interpret problems through their own lens in that domain. They often try to force their interpretation on decisions to address the problem leading to inappropriate solutions. In particular problems in the complex domains require a holistic dynamic approach that allows emergence rather than planning would benefit from an ecosystems perspective

Wicked problems such as climate change where the context is conceptualised as a ecosystem fall into the unordered Cynefin domains. Drawing on Complexity Theory and the characteristics of ecosystems which fit the Complex Cynefin domain described above, solutions to climate change problems should rely on the detection and leveraging of emergent patterns rather than ordered preplanning. This approach guides the analysis of Web 2.0 addressed in this paper. 


\section{A PRACTICAL APPLICATION OF ECOSYSTEMS CONCEPTS AND THE CYNEFIN}

\section{FRAMEWORK}

Ecosystems with their many interrelated and interconnected elements, are suitable constructs for representing complex unordered problems for which there are usually no obvious or straightforward solutions. However, just because ecosystems are complex does not mean, that they defy research analysis or practical application leading to constructive strategies for dealing with such problems. For example, knowledge management (KM), the field in which the Cynefin framework was developed, is a diverse, dynamic and amorphous topic, producing many wickedly complex problems and strongly conflicting opinions. This is demonstrated by the contradictory views of explicit and tacit knowledge that can be treated sometimes as a thing to be captured, stored and accessed, while at other times knowledge is treated as a flow to be shared among people, increasingly using the social technology in digital ecosystems. One of the pervasive elements that introduces such complexity into KM is the difficulty of assessing or measuring the value of KM projects in a meaningful way. A reason for this is that the objectives of KM initiatives are basically to improve organisational performance in ways that cannot easily be quantified. A further challenge is to find a direct link between the KM efforts and any such improvement. Without some means of measuring success it is then hard to justify the costs of potential KM projects to management. Benchmarking one organisation's KM program against another is also difficult as KM is so context dependent. It is simply not possible to transfer something that works in one organisation to another as the contextual elements and culture may not be receptive. Most KM programs include the need to create the necessary climate for change but this can take time. This complex interconnectedness, makes KM an ideal candidate topic where the use of the concept of an ecosystem can lead to creative solutions to wicked problems.

An instance of the use of the ecosystem construct is the Australian Knowledge Management (KM) Standard, the final version of which (AS5037-2005), relies on the concept of a 'knowledge ecosystem' to underpin a forward-looking representation of KM. Despite criticism and controversy (Australian KM Committee 2004, Hasan \& Lee 2004) this KM Standard broke new ground as an informed description of the current and emerging landscape in the area rather than a traditional prescriptive standard to be enforced by laws or regulations.

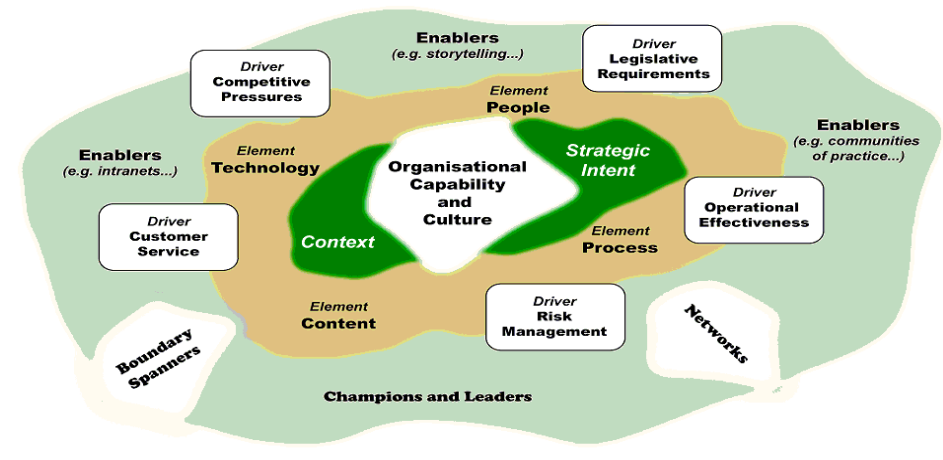

Figure 2 A visualisation of the Knowledge Ecosystem from the Australian KM Standard 
The development of the Australian KM Standard took place in three stages over a period of five years showing an evolution from an ordered linear approach in a handbook (HB275-2001) and the Interim Standard (AS5037-2003 \{INT\}) to a complex unordered perspective based on the knowledge ecosystem in the final version, AS5037-2005. Here the elements, enablers and other KM factors are conceptualised as a knowledge ecosystem as shown in Figure 2. This approach was strongly influenced by notions from the complexity quadrant of the Cynefin framework (Snowden 2002) where cause and effect cannot be predicted in advance and attractors and boundaries replace rules and control. Through the knowledge ecosystem, the Standard recognises that every KM initiative is different and unpredictable because of the unique context of each organisation. It also recognises that KM processes are organic and emergent rather than mechanistic and controlled.

The Standard does not promote a prescriptive, universal, linear KM process but rather a dynamic cyclic set of three phases:

mapping: an audit of the current organisational $\mathrm{KM}$ state in the local context and culture and identifying suitable KM goals,

building: the vital phase of prototyping, trialling projects, building trust, generating champions,

operationalising: the implementation of formal $\mathrm{KM}$ initiatives and programs including determination of effectiveness, measurements and performance evaluations.

The knowledge ecosystem expresses the pragmatic and practical interpretation of these concepts and where they fit into the Cynefin framework. The mapping process usually involves an ordered process of expert investigation and so is predominantly in the knowable Cynefin domain. The building phase of the KM process is one of exploration and experimentation to probe , sense and respond to the unique combination of organisms, physical and organisational environment and their many interrelated and interconnected elements. This reflects both the organic nature of an ecosystem and the typical ambiguous characteristics of the complex Cynefin domain. Typical of the unordered Cynefin domains, the Standard emphasises the importance of context and warns that what works in one organisation at one time might not be appropriate at others. The operationalising phase is an ordered process where formal KM systems mapped in the knowable domain, built in the complex domain, are implemented following established ordered procedures, typical of the known Cynefin domain. These activities reflect the dynamic and emergent nature of the local KM process.

In the following section of the paper we apply this combination of ecosystems theory and the Cynefin framework to another context, namely that of the contribution of Web 2.0 technologies to solving the wicked problem of climate change. An ecosystems approach such as that in the KM Standard can be used for initiatives in this cross disciplinary area where there is a need to include multiple levels of understanding of and action by the many interrelated and interconnected contextual elements, by representatives of local, national and international governments, business organisations and by consortia of academia,. We believe that social KM technologies have the potential to enable digital ecologies that will contribute to solutions to climate change by enabling human enterprises to reduce their carbon footprint through new ways of communication, coordination and cooperation. 


\section{CLIMATE CHANGE: A WICKED PROBLEM}

Environmental problems that threaten the very existence of the human race are arguably the most important issues of our time. There is a complex range of interrelated environmental issues that currently challenge decision-makers at local, national and international levels and our Australian experience is no different. Firstly there is the large body of scientific knowledge from many disciplines which is synthesized and interpreted by others according to their needs and biases. Secondly, there is the myriad of technological and engineering R\&D endeavours aiming at energy savings, clean energy generation, green urban design and so on. Thirdly, there are the logistical, business, political and informational issues that surround the science and engineering efforts and thus determine their acceptance, implementation and chances of success balanced against economic and social considerations. As championed by one of the most prominent IT companies over many years, IBM, the IT community has a major role to play in this third category: "As everything becomes more intelligent and interconnected, addressing energy and environment challenges requires innovative technology and deep business insight."2

Despite many decades of lobbying by scientists and environmental groups, climate change has only really captured the attention of national leaders over the past couple of years. A contributing factor has been the spread of network-centric advocacy supported by social technologies of Web 2.0. Advocacy groups on all sides of the debate are using ICT, the WWW and associated media to promote their causes. Climate change and environmental sustainability are issues where information and knowledge are vital, and social and cultural elements are critical. The capability of World Wide Web (WWW) to support the dissemination of information and knowledge is unprecedented, opening the door for an increased role for IS in addressing the climate change problem.

The increased role of ICT in the climate change debate is an emerging topic in the field of Information Systems (IS) where the term 'Green IS' is distinguished from 'Green IT'. In a new IS textbook, Boudreau et al (2008) record that 'Green IT' is seen to focus mainly on energy efficiency and equipment utilisation. 'Green IS', in contrast, refers to "the design and implementation of information systems that contribute to sustainability of business processes". The authors give examples such as reducing transportation costs, supporting teamwork and meetings, tracking environmental information, monitoring a firm's operational emissions and waste, and providing information to consumers. Green IS as so described should therefore have a greater potential than Green IT because it tackles a much larger problem. A more positive message supporting this position can be found in the work of Romm et al (1999) who noted at that time that the Internet economy was generating both structural and efficiency gains leading to emission reductions. Fuhr and Pociask (2007) recently reported on a study determining reduction in greenhouse emissions through the wide delivery of broadband services in the US and the work of Fernandez et al (2008) on how IS design can support and coordinate a project to extract oil from green algae. This message

\footnotetext{
${ }^{2}$ http://www-935.ibm.com/services/us/gts/html/services-green.html
} 
is driven home in the Smart 2020 project GeSI (2008) and a UN media release (UN 2007). However, literature in this area is scarce and there is certainly potential and a need for more.

The global nature of climate change and its global consequences also challenge IT and IS professionals. As IT and IS professionals we can continue to be introspective focussing our research effort on technologies and systems or take a responsible view as world citizens using our knowledge and skills outside our narrow discipline boundaries on something important to all who make up our global ecosystem. There is evidence that IS and IT professionals are becoming aware of these challenges with calls for submissions for special issues of journals, e.g. the Journal of Strategic Information Systems and Information and Organization and specialised tracks at the AMCIS and ICIS conferences this year.

\section{ICT, WEB 2.0, DIGITAL ECOSYSTEMS AND CLIMATE CHANGE}

Previous sections of the paper have presented, firstly, the ecosystem construct and the suitability of the Cynefin framework as a theoretical lens through which to make sense of an ecosystem and, secondly, a demonstration of a practical application of an ecosystem perspective in the Australian KM Standard. A wicked problem, climate change, has then been introduced as one where the Cynefin framework and the ecosystem perspective can be used to make sense of the place of digital ecosystems in a meaningful solution. A distinction was made above between the term Green IT, which sees ICT in a negative light as a major contributor to carbon emissions in their construction, use and disposal as waste, and Green IS which looks positively to ICT as a provider of solutions that reduce the carbon foot print of human enterprise. In this section of the paper we deal with the latter, briefly discussing some ordered solutions, and then, in more detail, solutions to climate change problems that reside in the unordered Cynefin domains and involve the use of digital ecosystems. We see ordered solutions consist mainly of systems that process information or regard knowledge as a thing. Unordered solutions are more likely to deal with a flow of knowledge for communication and collaboration at work and in society in general through Web 2.0 social technologies.

\section{Ordered activities: from the paperless office to e- and my- everything}

There are many straightforward uses of ICT that obviously and simply reduce carbon emissions and mitigate against climate change. Most routine human activity, located in the Cynefin ordered domains, has either been automated by an information system, such as an ERP, or is supported by standard ICT packages. Examples include web based services, reduction in paper-based storage of information and content knowledge, and carbon calculators.

Web services also have a positive environmental impact when online transactions replace the need for paper documents and the energy needed to move people to the shop-front. Trends in business and government are to electronic business, (e-commerce e-business e-government, e-health) where customers initiate, drive and manage transactions. This is not just a matter of getting the technical aspects right but motivating people to work and operate this way dat-to-day through winning over hearts and minds and confidence. For example, a sense of ownership and control comes through the prefix 'my'; I register my car at my-rta and choose TV programs through my-abc etc. This is becoming accepted widespread for simple ordered activities but is a challenge for unordered ones as will now be discussed. 
Climate change concerns suggest that more attention needs to be paid to the way these systems provide means of paperless creation, storage and availability of information and content knowledge. Curbing the urge to print hardcopy, we should encourage more use of digital document readers, digital editing capability (eg use the tracking and commenting facilities in wordprocessors), and online data collection, store, manipulation and display (eg online surveys).

Specific software such as carbon calculators can be used to forecast and monitoring the carbon emission from routine activity. Optimising systems to make manufacturing systems, logistics, supply chain etc more efficient and thus save energy, modelling business systems and processes to include environmental costs and benefits.

\section{Unordered activities - communicating, conferencing, coordinating, collaborating and advocating}

For any human enterprise to perform effectively, it needs to develop social capital as people meet, communicate and collaborate. Traditionally, people have preferred to meet face-to-face (F2F) and to have a daily routine where they 'go to work'. However we now recognize that such activities have a significant carbon footprint, travelling, commuting and producing of all manner of paper documentation. Potential IT solutions to these problems have been available for some time, namely teleconferencing, telecommuting, the virtual office, group decision support systems, and digital document management. Despite research showing their benefits, their take up has not been particularly widespread as people have resisted the combination of technical, economic, social and cultural changes to the way things are done.

Putting the ' $\mathrm{C}$ ' (communication) into ICT, together with the new imperative to take environmental concerns into account, brings the ICT-enabled conferencing and collaborating tools back firmly on the agenda. The challenges to IS are particularly acute now that social technologies are being considered as tools to support work in formal organisations whose culture is the antithesis of the type of ecosystem of communities who use these technologies socially. This development concerns knowledge as a flow which places it in the unordered Cynefin domain and poses a cultural challenge to organisations that can only be met with initiatives that suit the unordered domain and whose successful outcomes are judged accordingly (Hasan \& Crawford 2007).

Research in this area (Hasan 2005) takes a holistic approach to the complex issues of human activity in modern social-technical systems. The research integrates the technical, economic, social and cultural issues of working in the modern digitally networked world, understanding organisations as complex evolving ecosystems and finding ways to prepare managers and staff for a new and better way of working in this environment. Without this generation of new human capability and will, no amount of technical innovation is sustainable.

ICT can play a beneficial, environmentally friendly role in re-organizing and transforming the ways we work if an informed, balanced approach is taken. Findings from our research (Hasan et al 2007) suggest the following:

- form work teams and units of diverse members with complementary, not similar skills

- replace some F2F with online when appropriate and with suitable training.

- set up teams with F2F to build trust and social capital and meet again from time to time to celebrate success and renew team bonds.

- use appropriate groupware technologies under the guidance of those who know how, with moderators and facilitators, 
- build capability in virtual social skills, encourage community spirit

- develop ways to create, store and access information digitally

- allow/trust people to work/collaborate virtually in less formal ways, self-organized, selfdirected, letting team roles emerge.

- allow workers to use technologies of their own choosing

- provide appropriate incentives and rewards

The adoption of Web 2.0 in some organisations is happening under the label of Enterprise 2.0 although it is noticeable that organisations find it hard to break away from an ordered mode of implementation - restricting access, imposing structure, and strictly monitoring content. This is evident from the discussion by KM experts on $\mathrm{ActKM}^{3}$ on the use of Sharepoint in organisations for whom they consult and our research with Confluence $\bigodot^{4}$ as an organisational wiki (Hasan et al 2009). Hopefully management will learn from the way society at large is connecting in Web 2.0. Some enlightened companies are enlisting help from their customers and clients by creating digital ecosystems that allow both staff and their customers and clients to interact with a view to helping companies to improve their business, eg the online Lego user community proposes new designs for their product (Bauwens 2008), and $\mathrm{CNN}^{5}$ has instigated a program, i-report, where viewers supply news stories extending the operation of their business to the customer and client communities.

Digital ecosystems on the Web are also putting ordinary people in more direct contact with firsthand accounts of an event or with the multiple perspectives on issues of concern that allow them to generate their own opinion, and giving a strong collective public voice on environmental issues and climate change that is multidisciplinary and cross cultural, with different languages and jargon and foci. Supported by Web 2.0, the balance of power with respect to knowledge is now shifting from the 'official versions' in the hands of governments, big business, media moguls, formal libraries and publishing houses. Now if anyone wants to 'know' they are more likely to go to Google ${ }^{6}$ or Wikipedia ${ }^{7}$. Many, particularly young, consumers of news are cynical about what they read in newspapers or see on television. This has contributed to the decline of newspapers sales. They read blogs from people on the scene, discuss current events on Twitter, get personal opinions from postings on Myspace or Facebook, become immerse in virtual worlds on Second Life, see pictures on Flickr or videos on Youtube, often with detail of news stories before they are picked up by the traditional news media. A list of many of these 'social networking' sites, together with a brief description of their focus is available in Wikipedia ${ }^{8}$.

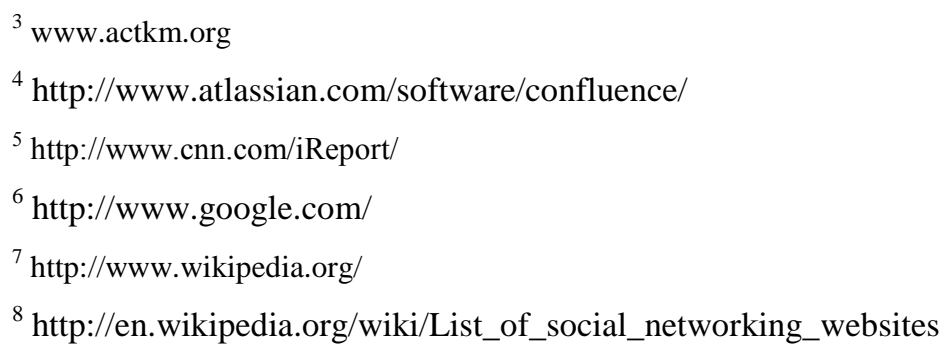


These social networking applications have had the effect of democratising knowledge within organisations (Hasan \& Pfaff 2007) and in society in general (Paulussen et al 2007) where they enable a form of network-centric advocacy which is changing the political landscape. Voters can expose themselves to new perspectives on issues and collaborate with others to get new messages 'out there', see for example in Australian the independent news site Crikey ${ }^{9}$ and the community advocacy organisation Getup ${ }^{10}$. This phenomenon is almost certainly helping the environmental movement with knowledge sharing and network-centric advocacy in an unordered but influential fashion.

\section{DISCUSSION AND CONCLUSION}

In this paper the Cynefin framework was presented as a theoretical lens through which to make sense of the diversity within ecosystems, including those which present as wicked problems. The example was given of the application of the Cynefin framework to the ecosystems construct in the Australian KM Standard. Climate change is sensibly viewed as a wicked problem to be interpreted through the lenses of Cynefin and the ecosystems construct. We propose that the IS discipline can contribute to the solution of these wicked problems. Technical and social aspects of Web 2.0 are interrelated elements in the threatened ecosystems within which we now live as individuals and as IS professionals. The capability that online social technologies provide to support the coordination of dispersed human enterprises has obvious potential benefits to the cost of these enterprises, to social wellbeing, and to environmental impact. This perspective has implications not only for the practical decisions and action we take in response to the climate change challenge but also the way we conduct research that will produce meaningful results that will inform these decisions and actions.

We argue that a holistic, realistic and sensible perspective is needed for research into wicked problems, a perspective that will produce results that will make a difference in practice. In this paper we propose that the Cynefin sense-making framework provides the breadth of view to underpin such research where the problem is seen to be situated within a dynamic ecosystem comprising technological tools, social and contextual elements. Furthermore, we propose that taking this approach to the incorporation of Web 2.0 into a solution to the climate change problem is an instance of the way the IS/IT community can remain relevant to the real world and be seen to make a significant contribute in a time of global crisis.

An appropriate research agenda for IS would be to study the development of Web 2.0 from the holistic perspective of purposeful human activities within the ecosystem context. In some cases, particularly in large formal human enterprises, this is planned and ordered in the same way as most organisational information systems. However, Web 2.0 is really a phenomenon that has arisen in the chaotic social world where activities are not planned and so in most cases they work best in the

\footnotetext{
${ }^{9}$ http://www.crikey.com.au/

${ }^{10} \mathrm{http}: / / w w w . g e t u p . o r g . a u /$
} 
context of a complex ecosystem. This context requires an understanding of the way things work in the complex domain of the Cynefin framework and many organisations have ordered cultures that often do not suit this. One factor to consider is that, while it is probably appropriate to see current developments as being driven by the technology, it is fair to say that Web 2.0 can be better viewed as a strong enabler of new ways of conducting collaborative human activities that have cost, social and environmental benefits.

Future research should look at typical collective human activities, their purpose, anticipated outcomes and the ways they have always been done. IS researchers could then investigate how the various aspects of Web 2.0 are, or could be, incorporated and how the environmental impact of different ways of carrying out these activities can be anticipated, compared and assessed.. We believe that it makes no sense to look at the activities or the technologies in isolation but that investigating how the various elements interact and what consequences, both intended and unintended, could result from interventions in the conduct of those activities will be of benefit. There would be little point, for example, in replacing a F2F meeting with virtual one, if the purpose of the meeting was to develop interpersonal relationships and this objective was not achieved in the virtual environment. On the other hand if a group of employees were developing good interpersonal relationships using a Web 2.0 application that was not officially sanctioned by the company, this should not be immediately treated with suspicion by management, as is often the case, but rather it should be investigated cooperatively with the employees for possible changes to the rules relating to the use of such applications: Would the company be prepared to take the risk that this might have undesirable consequences in misuse of the system or open the flood to requests for access to other Web 2.0 applications? Is there a greater risk in restricting use of social technologies in the workplace that employees use ubiquitously in the rest of their lives? How can the use of this application enable the organisation to achieve cost, social and environmental benefits?

Framing research by means of the Cynefin domains enables a researcher to separate out topics that are knowable where more traditional research approaches can be used from those wicked problems that are complex where more innovative approaches to data collection and analysis are needed. It is important to find methods appropriate to conducting research into complex issues. Some that we have used include: systems modelling (Hasan 2007), emergent design research (Hasan 2005), simulation (Hasan et al 2009) and gaming (Crawford \& Hasan 2007). For problems in the complex domain the notion of an ecosystem can then be used to conceptualise the current environment in a holistic, dynamic way that is appropriate for the study of wicked problems and facilitates the finding of innovative solutions. Only by being creative in our thinking and approaches to research can the critical problems of climate change and possible Web 2.0 solutions be understood and advanced.

\section{REFERENCES}

ACS (2007) ACS Policy for Green IT available at http://www.acs.org.au/acs_policies/docs/2007/greenictpolicy.pdf

AS5037-2003 \{INT $\}$ Interim Australian Standard Knowledge Management, Standards Australia, 2003.

AS5037-2005 (2005) Australian Standard Knowledge Management, Standards Australia,.

Australian KM Committee (2004) KM Standards: developments in Australia, Journal of Knowledge Management Research and Practice, 2, pp.58-60. 
Bauwens M (2008) Lego's participatory army marches on, http://www.experienceeconomy.com/2008/04/25/

Boudreau M. Chen A. Huber M. (2008) Green IS: Building Sustainable Business Practices a chapter in Richard Watson ed. Information Systems: a Global Text project http://globaltext.terry.uga.edu/

Courtney J.F. (2001). Decision making and knowledge management in inquiring organizations: toward a new decision-making paradigm for DSS. Decision Support Systems 31, 17-38

Crawford K. Hasan H. (2007) Network-Centric Characteristics in Organizations: The Application of Gaming, Proceedings of UKAIS2007, Manchester.

Fernandez W. Bergvall-Kareborn B. Djordjevic M. Lovegrove K. Fernandez Velasco J. Talent M. (2008) How IS Design can Contribute to major Climate Mitigation Projects, Proceedings of the ISF Conference, Canberra.

Fuhr J Pociask S. (2007) Broadband Services: Economic and Environmental Benefits a paper for the American Consumer Institute, available at http://www.aci-citizenresearch.org

Garnaut Climate Change Review Final Report (2008) available at http://www.garnautreview.org.au/domino/Web_Notes/Garnaut/garnautweb.nsf

GeSI (2008) SMART 2020 Report by GeSI and The Climate Group, available at http://www.gesi.org/

Ghose A, Hasan H. Spedding T.(2008) Carbon-Centric Computing: IT Solutions for Climate Change. CCCI Report, http://www.uow.edu.au/ aditya/research/ccci/

Gore A (2006) An Inconvenient Truth DVD and companion book, An Inconvenient Truth: The Planetary Emergency of Global Warming and What We Can Do About It,

Hasan H (2007) Systems Dynamics Modelling of Human and Information Aspects of NetworkCentric Configurations, Proceedings of MILCIS, Canberra

Hasan H. (2006) Design as Research: Emergent Complex Activity, Proceedings of the Australasian Conference of Information Systems, Adelaide, Dec 2006

Hasan H. (2005) Socio-Technical Systems: from individual transaction to situated community activity, in G. Whymark and H. Hasan (eds) Activity as the Focus of Information Systems Research, Knowledge Creation Press, UCQ, 29-46.

Hasan H. Crawford K (2007) Knowledge Mobilisation in Communities through Socio-Technical System, Journal of Knowledge Management Research and Practice 5/4 237-248.

Hasan H. Lee V. (2004) Managing Knowledge as a Strategic Business Asset, ISO Management Systems, International Review of ISO9000 and ISO 140000, , 4/2 pp. 37-39.

Hasan H. Meloche J. Alkayid K (2009) Simulating Information Exchanges to Investigate the Utility of Public Health Websites, accepted for Transformation Government: People, Process and Policy: ICT in Public Sector

Hasan H., Meloche J. Pfaff C. Qi Y. Willis D. (2009) Co-creating Corporate Knowledge with a Wiki, International Journal of Knowledge Management, 5/2 33-50.

Hasan H. Pfaff C (2007) Democratising Organisational Knowledge: the Potential of the Corporate Wiki, Proceedings of the International Conference of Information Systems (ICIS 2007) Montreal 
Hasan H. Warne L. Linger H. (2007) The Sensible Organization: A new agenda for IS research, Proceedings of the International Conference of Information Systems (ICIS 2007) Montreal

HB275-2001 Knowledge Management: A Framework for succeeding in the knowledge era, Standards Australia,

Kurtz, C. F.; Snowden, D. J. (2003) The New Dynamics of Strategy: sense-making in a complexcomplicated world, IBM Systems Journal, Fall 2003

Paulussen, S., Heinonen, A., Domingo, D. and Quandt, T. (2007) Doing It Together: Citizen Participation in the Professional News Making Process, Observatorio (OBS*) Journal, 1(3), pp.131-154

Rittel, H., Webber, M. (1975). Dilemmas in a General Theory of Planning. Policy Sciences 4, 155169

Romm J Rosenfeld A. Herrmann S. (1999) The Internet Economy and Global Warming; A scenario of the Impact of E-Commerce on Energy and the Environment, The Center for Energy and Climate Change Solutions, see http://222.cool-companies.org

Snowden, D. (2002) Complex Acts of Knowing: Paradox and Descriptive Self-Awareness. Journal of KM, 6(2).AS5037(Int) Interim Australian Standard Knowledge Management, Standards Australia, 2003.

Stern Review (2006) The Economics of Climate Change, available at http://www.hmtreasury.gov.uk/independent_reviews/stern_review_economics_climate_change/stern_revie w_Report.cfm

UN (2007) Information technology can be harnessed against climate change, available at http://www.un.org/apps/news/printnewsAr.asp?nid=24815

Von Bertalanffy V (1967) Robots, Men, and Minds; Psychology in the Modern World. New York: Braziller. 